\title{
When birth is not as expected: a systematic review of the impact of a mismatch between expectations and experiences
}

Rebecca Webb ${ }^{1 *}$, Susan Ayers ${ }^{1}$, Annick Bogaerts ${ }^{2,3,4}$, Ljiljana Jeličić 5,6 , Paulina Pawlicka7 , Sarah Van Haeken ${ }^{2,8}$, Nazihah Uddin ${ }^{1}$, Rita Borg Xuereb' ${ }^{9}$, Natalija Kolesnikova ${ }^{1}$ and COST action CA18211:DEVoTION team

\begin{abstract}
Background: Pregnancy and childbirth are significant events in women's lives and most women have expectations or plans for how they hope their labour and birth will go. It is possible that strong expectations about labour and birth lead to dissatisfaction or other negative outcomes if these expectations are not met, but it is not clear if this is the case. The aim was therefore to synthesise prospective studies in order to understand whether unmet birth expectations are associated with adverse outcomes for women, their partners and their infants.

Method: Searches were carried out in Academic Search Complete; CINAHL; Medline; PsycINFO, PsychArticles, PubMed, SCOPUS and Web of Science. Forward and backward searches were also completed. Studies were included if they reported prospective empirical research that examined the association between a mismatch in birth expectations/ experience and postnatal outcomes in women, their children and/or their partners. Data were synthesised qualitatively using a narrative approach where study characteristics, context and methodological quality were extracted and summarised and then the differences and similarities among studies were used to draw conclusions.

Results: Eleven quantitative studies were identified for inclusion from nine countries. A mismatch between birth expectations and experiences was associated with reduced birth satisfaction. Three studies found a link between a mismatch and the development of postnatal post-traumatic stress disorder (PTSD). The evidence was inconsistent for postnatal depression, and fear of childbirth. Only one study looked at physical outcomes in the form of health-related quality of life.

Conclusions: A mismatch between birth expectations and experiences is associated with birth satisfaction and it may increase the risk of developing postnatal PTSD. However, it is not clear whether a mismatch is associated with other postnatal mental health conditions. Further prospective research is needed to examine gaps in knowledge and provide standardised methods of measuring childbirth expectations-experiences mismatch. To ensure women's expectations are met, and therefore experience a satisfying birth experience, maternity providers should provide sensitive care, which acknowledges women's needs and preferences, is based on open and clear communication, is delivered as early in pregnancy as possible, and enables women to make their own decisions about care.
\end{abstract}

Trial registration: Protocol registration: PROSPERO CRD42020191081.

Keywords: Birth expectations, Birth experiences, Birth plans, Mismatch, Psychological outcomes, Birth satisfaction

\footnotetext{
* Correspondence: Rebecca.Webb.2@city.ac.uk

${ }^{1}$ Centre for Maternal and Child Health Research, City, University of London, London EC1V OHB, UK

Full list of author information is available at the end of the article
}

(c) The Author(s). 2021 Open Access This article is licensed under a Creative Commons Attribution 4.0 International License, which permits use, sharing, adaptation, distribution and reproduction in any medium or format, as long as you give appropriate credit to the original author(s) and the source, provide a link to the Creative Commons licence, and indicate if changes were made. The images or other third party material in this article are included in the article's Creative Commons licence, unless indicated otherwise in a credit line to the material. If material is not included in the article's Creative Commons licence and your intended use is not permitted by statutory regulation or exceeds the permitted use, you will need to obtain permission directly from the copyright holder. To view a copy of this licence, visit http://creativecommons.org/licenses/by/4.0/. The Creative Commons Public Domain Dedication waiver (http://creativecommons.org/publicdomain/zero/1.0/) applies to the data made available in this article, unless otherwise stated in a credit line to the data. 


\section{The impact of a mismatch between expectations and experiences of birth on postnatal outcomes: A systematic review.}

Pregnancy and childbirth are significant events in women's lives that can be associated with both positive and negative emotions. Research suggests that childbirth may affect a woman's sense of self [1,2], and her physical and psychological wellbeing [3, 4]. A positive birth experience can provide women with feelings of satisfaction and empowerment $[5,6]$, whereas a negative birth experience can lead to feelings of disappointment [7], the delay of subsequent pregnancies [8], and in some cases the development of postnatal psychological difficulties such as post-traumatic stress disorder (PTSD) $[9,10]$ or depression [11], which are in turn associated with poor offspring developmental and psychological outcomes [12-23].

Pregnant women may form beliefs about what to expect during labour and birth. These expectations may be developed from antenatal education, books, television, the internet, healthcare providers or family and friends [24-29]. Many expectations about birth may also be in line with the belief system a woman holds about birth. One model, (the technocratic model) views childbirth as inherently risky, with the foetus being separate from the mother, the health of the infant during pregnancy and labour needing to be ensured through tests, and medication, and birth needing to be completed within a "safe" time window [30]. On the other hand, the holistic model of birth sees the mother and foetus as one, labour being a normal physiological process which generally does not require intervention and that happens at its own rate [30]. The development of these belief systems are likely to come from a range of sources, including previous birth experiences [31], and are often associated with the type of birth a woman may choose [32, 33].

Based on their expectations and beliefs, some women create specific birth plans, which may include preferences for how each stage of labour should be managed, pain relief options and immediate postnatal care, such as skin to skin contact [34]. However, the development and use of birth plans is a contentious issue for both women and healthcare professionals. For example, an analysis of online UK parenting forums found that some women believe that the idea of planning birth is problematic because labour and birth can be unpredictable [35]. Further, a qualitative study of nine maternity professionals found that midwives believe the term "birth plan" can be misleading, and may contribute to women having unrealistic expectations during labour [36]. Additionally, a qualitative study of women and healthcare professionals found that respondents believed having strong expectations and preferences during labour may lead to disappointment or dissatisfaction if these expectations are not met [37]. However, some of the respondents from these studies believed that clear birth plans or expectations can be used as a tool for communication between women and healthcare professionals and can therefore be beneficial [35, 37].

Cross-sectional research supports the idea that unmet birth expectations can affect birth satisfaction. For example, a survey of 442 postnatal women found that those whose birth plans were followed had significantly higher birth satisfaction levels [38]. On the other hand, a survey of over 2500 women found that those who had unexpected medical complications during birth were more likely to rate their childbirth experience as negative [39]. Qualitative data also supports these findings. For example, one study found that for women to perceive their birth as positive, they had to achieve their birth expectations [40]. Similarly, qualitative data from 115 women identified that where care had gone beyond expectations, birth satisfaction was increased [41]. However, as birth expectations in these studies were measured after women had given birth the results may be subject to recall bias and may not give an accurate representation of women's expectations prior to birth, making conclusions difficult to draw.

The summarised research gives an unclear picture about whether having clear birth expectations can be beneficial to women's birth experiences in terms of improved communication [35, 37], or lead to dissatisfaction or disappointment if expectations are not met [38-41]. Furthermore, many of the studies are cross-sectional surveys, where birth expectations are measured after women have given birth. These studies may therefore be subject to recall bias, and results may differ if women are asked about their birth expectations prior to giving birth and their experience after birth, so a prospective measure of any mismatch could be calculated.

Given the adverse impact a negative birth experience can have on women and their infants' [7, 9-11, 19-21], it is important for us to understand whether having unmet birth expectations can contribute to negative birth experiences, and in turn, if these impact postnatal outcomes. Understanding the impact of unmet birth expectations on postnatal outcomes (such as satisfaction, mental health, physical wellbeing etc.) could help to identify problem areas that should be improved [42] and therefore lead to the improvement of maternity care for women and their partners. Therefore, the aims of this literature review are to: 1) review the evidence on the impact of a mismatch between birth expectations and experience on postnatal outcomes for the mother, her partner and her infant using prospective studies, and 2) provide a critical appraisal and overview of the evidence base. 
Method

Protocol and registration

The protocol was registered with PROSPERO (CRD42020191081).

\section{Information sources and search}

Literature searches and study selection were reported according to the Preferred Reporting Items for Systematic Reviews and Meta-analyses (PRISMA) guidelines [43]. Online databases were used to identify papers. Boolean operators
(AND/OR) were used to combine subject headings and relevant search terms (see Table 1). Searches were limited to key words being present in the title of papers. The date of the last search was 6th April 2020. Forward and backward searches of included studies were carried out and completed by the 25 th June 2020 .

\section{Study selection}

Search results were imported into Eppi-Reviewer 4, where duplicates were removed and results were screened

Table 1 Inclusion criteria and search strategy

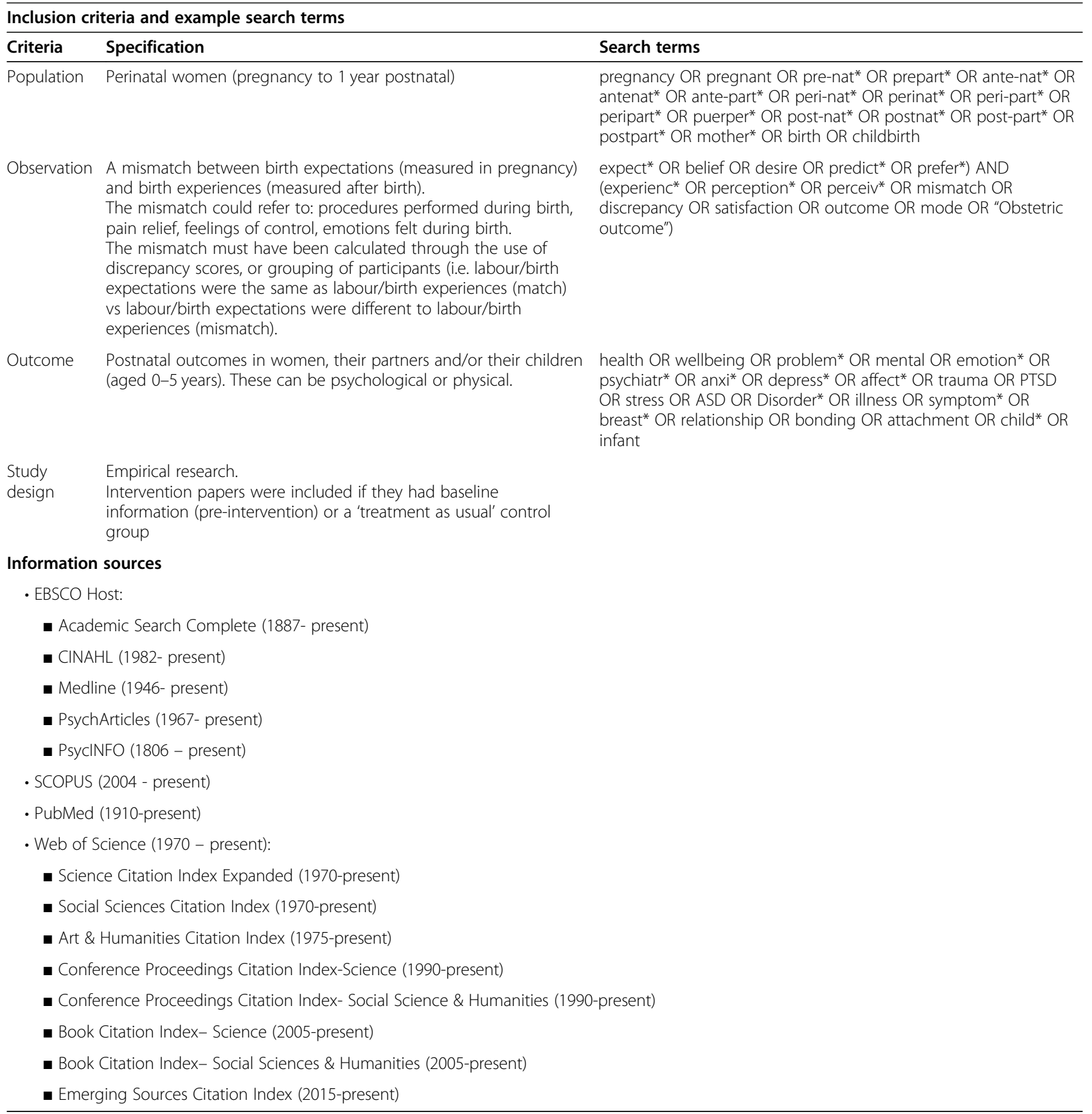


by title and abstract by NU based on inclusion criteria (see Table 1). A proportion (10\%) of the results were double screened by PP. Decisions to include or exclude were concordant between reviewers in $97.5 \%$ of cases. Once title and abstract screening was complete, full text screening was carried out by RW. A proportion (10\%) were double screened by PP and decisions to include or exclude were concordant between reviewers in 100\% of cases. Disagreements for both title and abstract and full text screening were discussed and resolved by NU, PP, SA and RW.

\section{Data collection process and data items}

Data extraction was carried out using Eppi-Reviewer 4 which allows for line-by-line coding. A new 'codeset' labelled 'Data Extraction' was created and contained every item to be extracted from the data (e.g. year of publication, country of study). Each study was read in full, and relevant parts of the text highlighted (for example the country of the study) and applied to the relevant code.

Data were extracted for the following domains: Study Characteristics (country, setting, design, aim); Sample Characteristics (recruitment, size, age, ethnicity, employment, education, children, socioeconomic status, mental health problems, obstetric details, other demographic details); Data collection (method, ethical approval; measure of mismatch; measure of postnatal outcomes; measure of other variables); and Results (mismatch; postnatal outcomes; other variables).

\section{Quality appraisal of individual studies}

Methodology sections of included texts were assessed for quality using Joanna Briggs Critical Appraisal Tools for Cohort studies [44]. Each point on the checklists can be coded into $\mathrm{Yes} / \mathrm{No} / \mathrm{Unclear} / \mathrm{Not}$ applicable. Items which were coded as 'Yes' were assigned a score of 1 , items coded as 'No' or 'Unclear' were assigned a score of 0 . The higher the score, the higher the quality of the study. Methodological assessment of studies was done by two raters: RW and SVH. Coders assigned the same score to studies $44.4 \%$ of the time. Most disagreements were due to one study [34], where there were disagreements on 6 out of the 9 items. The corresponding author of this study was contacted in September 2020 for further clarification of the methodology; however, no response was received before submission of the systematic review. When this study was removed from agreement calculations, coders assigned the same score to study 90.3\% of the time. All disagreements were discussed, with particular attention paid to Mei et al. (2016) [34] and were resolved by RW and SVH. The final appraisal is based on agreed answers. See supporting information for more details.

\section{Synthesis of results}

Studies were synthesised narratively by RW using the technique described by Lucas et al., (2007) [45]. First, study characteristics, context and methodological quality were extracted and summarised. Next, the differences and similarities among studies were used to draw conclusions across the studies. Due to the small number of included studies, and the heterogeneity of these studies, there was not enough data to conduct a meta-analysis.

\section{Results}

\section{Study characteristics}

Searches identified a total of 3684 citations. Hand searches of reference lists of included studies identified a further 2 studies. After removing duplicates and screening through abstracts, titles and full texts, 11 studies remained for inclusion in the review (Fig. 1). Studies included in the review are summarised in Tables 2 and 3. Half of the studies were carried out in English-speaking countries (Ireland $n=1$; Canada $n=2$, USA $n=4$ ). One study [46] was carried out in multiple countries (Germany, Ireland and Italy). Sample sizes ranged from 30 to 1700 with an average of 401 participants. Six studies reported the average age of women as being between 25 and 32.21. Three studies reported that over half of women (63.8-83\%) were aged between 26 and 35 . Of the four studies that reported ethnicity, women were mainly white (47.5-95\%) or Jewish (98.5\%). The samples were well educated with most women having received $12+$ years of education (54-96\%). Nine of the studies were published within the last 10 years (Range: 19822020; Mean $=2011$, Median $=2014$ ).

\section{Risk of bias}

Due to the lack of research in this area, an inclusive approach was taken, with no studies being excluded due to their quality. Nine out of the 11 studies were of good quality (scoring 7 out of 9 or more). One study was of medium quality (scoring 6) and one was low quality (scoring 3).

\section{Measurement of mismatch}

Three studies [46-48] used women's desired mode of birth and actual mode of birth to group women into matched vs mismatched groups (e.g. wanted a vaginal birth and had a vaginal birth = match vs wanted a vaginal birth and had a c-section = mismatch). One study [49] measured different aspects of labour and birth (e.g. medication, presence of family) and grouped women into four groups (1. fulfilled expectations - women expected an event to happen and it did happen; 2. unmet expectations - women expected an event to happen, but it did not happen; 3. unexpected experiences - women did not expect an event to happen but it did, and 4. null 


\section{श्रISMA PRISMA 2009 Flow Diagram}

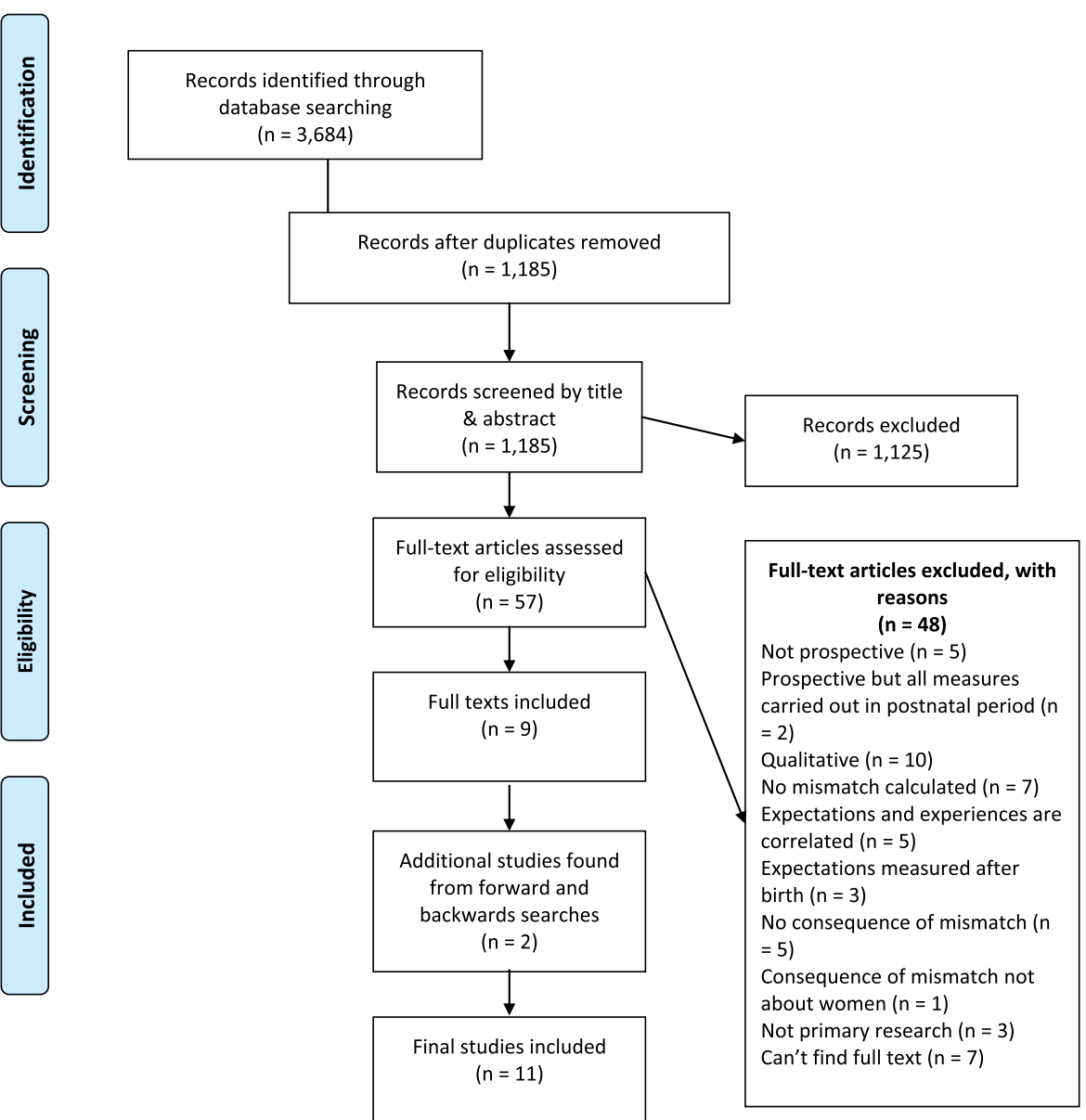

Fig. 1 PRISMA Flow Diagram

experiences - women did not expect an event to happen, and it did not happen). Another study [50] used a score to calculate women's preference for a vaginal birth $(0=$ no preference, $1=$ strong preference $)$ and used this variable to assess the interaction with actual mode of birth on the outcome measure. One study [34] calculated the percentage of expectations fulfilled based on women's own birth plans and their medical records. Two studies [51, 52] used the Wijma Delivery Expectancy Questionnaire (WDEQ-A [53]) to measure birth expectations and the Wijma Delivery Experiences Questionnaire (WDEQ-B [53]) to measure birth experiences. A difference score was calculated between the two where a negative score represented an experience that was more negative than expectations and positive scores revealed a more positive experience. The remaining studies $[54,55]$ calculated a discrepancy score based on what women wanted to happen during labour vs what actually happened or women's expectations of control during labour and experiences of control during labour [56].

\section{Outcomes of mismatch}

The outcomes of the mismatch were all measured for women. These were grouped into birth satisfaction $(n=2)$; psychological outcomes $(n=6)$; birth satisfaction and psychological outcomes $(n=2)$; and physical health $(n=1)$. No studies looked at the impact of a mismatch on women's partners or baby/children.

\section{Birth satisfaction}

Four studies looked at the impact of a mismatch between birth expectations and experiences on satisfaction with birth. Results suggest that if a woman had her birth expectations met, she was more likely to rate her birth 
Table 2 Study characteristics of included studies

\begin{tabular}{|c|c|c|c|c|c|c|}
\hline$\#$ & Author \& Year & $\begin{array}{l}\text { Country } \\
\text { of study }\end{array}$ & Study design & $\begin{array}{l}\text { Sample } \\
\text { size }\end{array}$ & Participant characteristics & Quality rating \\
\hline & Health related quality & of life $(n=1)$ & & & & \\
\hline \multirow[t]{2}{*}{1} & $\begin{array}{l}\text { Fobelets et al. (2019) } \\
\text { [1] }\end{array}$ & $\begin{array}{l}\text { Germany, } \\
\text { Ireland, Italy }\end{array}$ & $\begin{array}{l}\text { Prospective longitudinal } \\
\text { (part of RCT called OPTIBIRTH [2]) }\end{array}$ & 862 & $\begin{array}{l}\text { All women had previous C-section; } 83 \% \text { aged } \\
31 \text { or over; } 97 \% \text { married or cohabiting; 38\% } \\
\text { birthing in Germany, 15\% birthing in 47\% } \\
\text { birthing in Italy. }\end{array}$ & Good (Score: 7/9) \\
\hline & \multicolumn{6}{|c|}{ Psychological outcomes $(n=6)$} \\
\hline 2 & $\begin{array}{l}\text { Garthus-Niegel et al. } \\
\text { (2014) [3] }\end{array}$ & Norway & Prospective cohort & 1700 & $\begin{array}{l}\text { Age } M=31.2 ; 97.8 \% \text { married or co-habiting; } \\
47.2 \% \text { first time mothers }\end{array}$ & Good (Score: 8/9) \\
\hline 3 & $\begin{array}{l}\text { Houston et al. (2015) } \\
\text { [4] }\end{array}$ & USA & Prospective longitudinal & 160 & $\begin{array}{l}67.5 \% \text { aged over } 30 ; 47.5 \% \text { White; } 26.3 \% \\
\text { Black; } 33.1 \% \text { nulliparous; } 30.6 \% \text { previous } \\
\text { c-section }\end{array}$ & Good (Score: 8/9) \\
\hline 4 & $\begin{array}{l}\text { Philipson-Price } \\
\text { (1982) [5] }\end{array}$ & Canada & Cohort & 30 & Age $M=27.5$ & Medium (Score: 6/9) \\
\hline 5 & Soet et al. (2003) [6] & USA & Prospective cohort & 103 & $\begin{array}{l}\text { Mean age }=29.20(S D=4.99) ; 83 \% \\
\text { married; } 67 \% \text { white }\end{array}$ & Good (Score: 9/9) \\
\hline 6 & Sluijs et al. (2020) [7] & $\begin{array}{l}\text { The } \\
\text { Netherlands }\end{array}$ & Prospective cohort & 463 & $\begin{array}{l}\text { Most women }(63.8-74 \%) \text { aged between } 26 \\
\text { and } 35 \text { years old; } 98.2-98.3 \% \text { of women } \\
\text { married or cohabiting }\end{array}$ & Good (Score: 9/9) \\
\hline \multirow[t]{2}{*}{7} & $\begin{array}{l}\text { Verreault et al. (2012) } \\
\text { [8] }\end{array}$ & Canada & Prospective cohort & 308 & Age $M=32.21(S D=4.40) ; 97.1 \%$ married & Good (Score: 9/9) \\
\hline & \multicolumn{6}{|c|}{ Psychological outcomes and birth satisfaction $(n=2)$} \\
\hline 8 & $\begin{array}{l}\text { Stein De Luca \& } \\
\text { Lobel (2014) [9] }\end{array}$ & USA & Prospective longitudinal & 164 & $\begin{array}{l}\text { Age } M=30 ; 95 \% \text { White; } 97 \% \text { married or } \\
\text { cohabiting; } 23 \% \text { previously given birth }\end{array}$ & Good (Score: 8/9) \\
\hline \multirow[t]{2}{*}{9} & $\begin{array}{l}\text { Tanglakmankhong } \\
\text { (2010) [10] }\end{array}$ & Thailand & Prospective longitudinal & 195 & Age $M=25 ; 51.8 \%$ first time pregnancy & Good (Score: 8/9) \\
\hline & \multicolumn{6}{|c|}{ Birth satisfaction $(n=2)$} \\
\hline 10 & Mei et al. (2016) [11] & USA & Prospective cohort & 94 & $N R$ & Poor (Score: 3/9) \\
\hline 11 & Preis et al. (2019) [12] & Israel & Prospective longitudinal & 330 & $\begin{array}{l}\text { Age } M=30,98.5 \% \text { Jewish, } 95.2 \% \text { married } \\
\text { or cohabiting }\end{array}$ & Good (Score: 8/9) \\
\hline
\end{tabular}

experience as satisfying [34, 49]. Where birth expectations were not met childbirth satisfaction was low [55, 56]. As well as a direct relationship between a mismatch and childbirth satisfaction, one study [55] found an indirect relationship between a mismatch and childbirth satisfaction which was mediated by women's perceptions of control, both negative and positive emotions and women's perceptions of care. Furthermore, another study [49] showed that self-efficacy expectancy, childbirth class attendance and women's education levels were also predictors of childbirth satisfaction. Additionally, one study found that birth plans with a lot of requests were associated with an $80 \%$ reduction in birth satisfaction [34].

\section{Psychological outcomes}

Eight studies looked at the impact of a mismatch between birth expectations and experiences on psychological outcomes. Three studies looked at the impact of a mismatch on postnatal depression. Two studies found that a mismatch between birth expectations and experiences were not associated with postnatal depression symptoms $[54,56]$. On the other hand, one study found an association between a mismatch in birth expectations/experiences and postnatal depressive symptoms [50]. Results indicated that in women who had a caesarean section (CS), higher preference for a vaginal birth was associated with higher postnatal depression scores. This suggests other factors are likely to be more important in postnatal depression than a mismatch between birth expectations and experiences. This is supported by another study [56] that showed prenatal depressed mood, childcare stress, satisfaction with social support received from their partner during birth, concerns about the self and the foetus during pregnancy, and perceived control during birth were associated with postnatal depressed mood whereas a mismatch was not.

Two studies looked at the impact of a birth expectations/experiences mismatch on fear of childbirth (FOC), again with mixed results. One study [49] found that FOC (measured after birth) was associated with a mismatch (women did not expect the event to happen but it did) but this relationship was no longer significant after controlling for parity and complications during labour. On the other hand, the other study [48] identified that women who wanted a vaginal birth but had a CS were at 


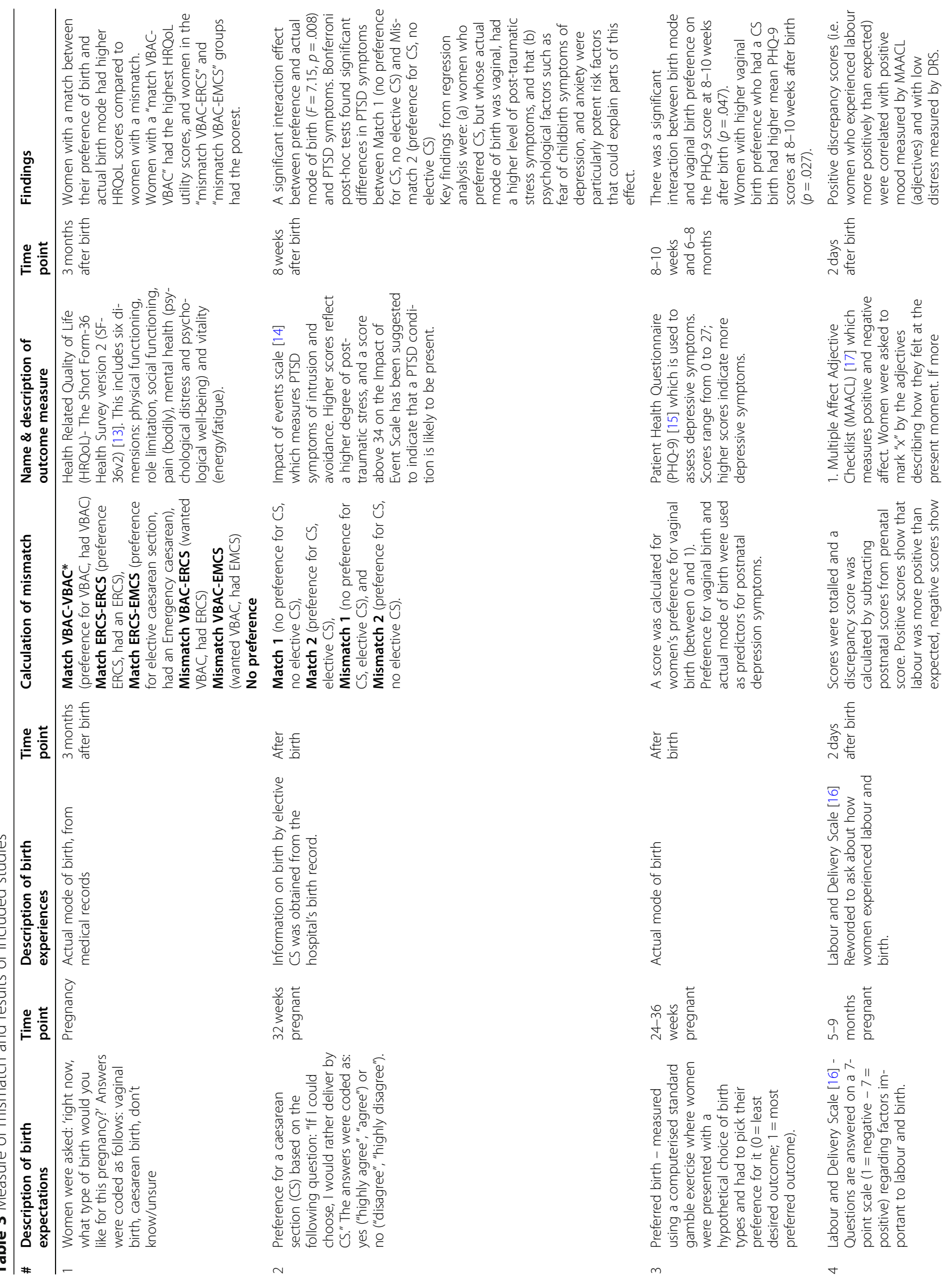




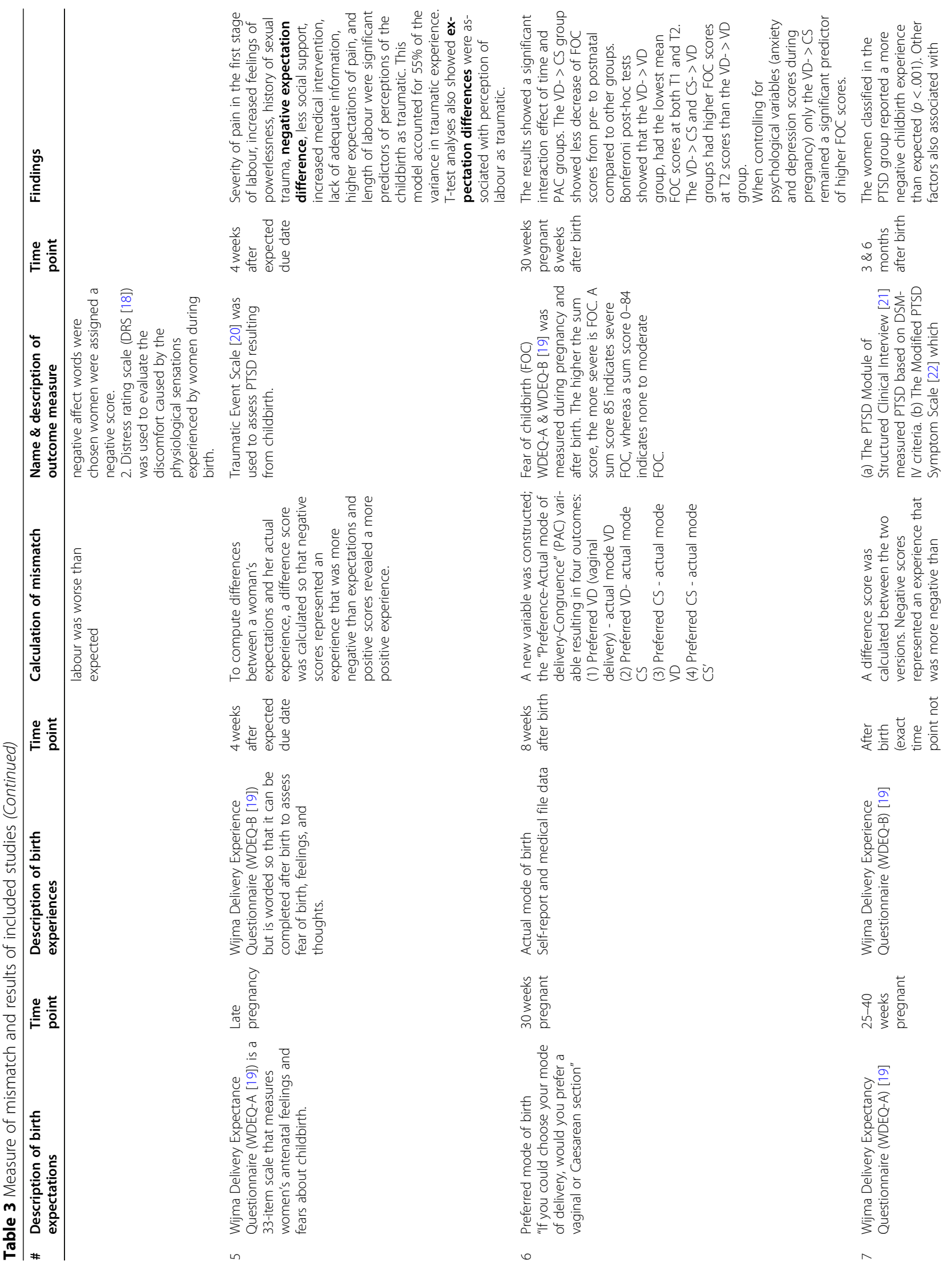




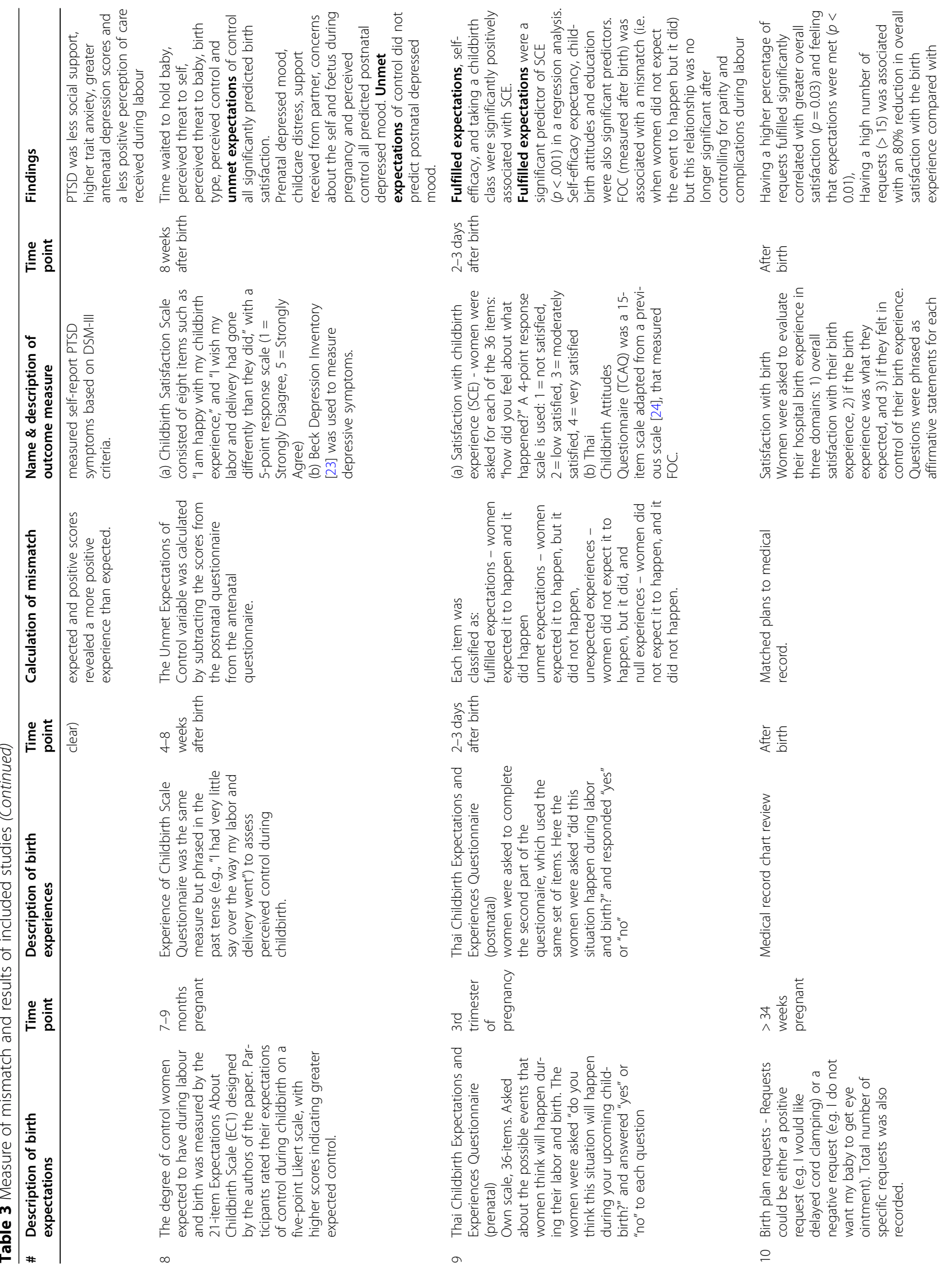




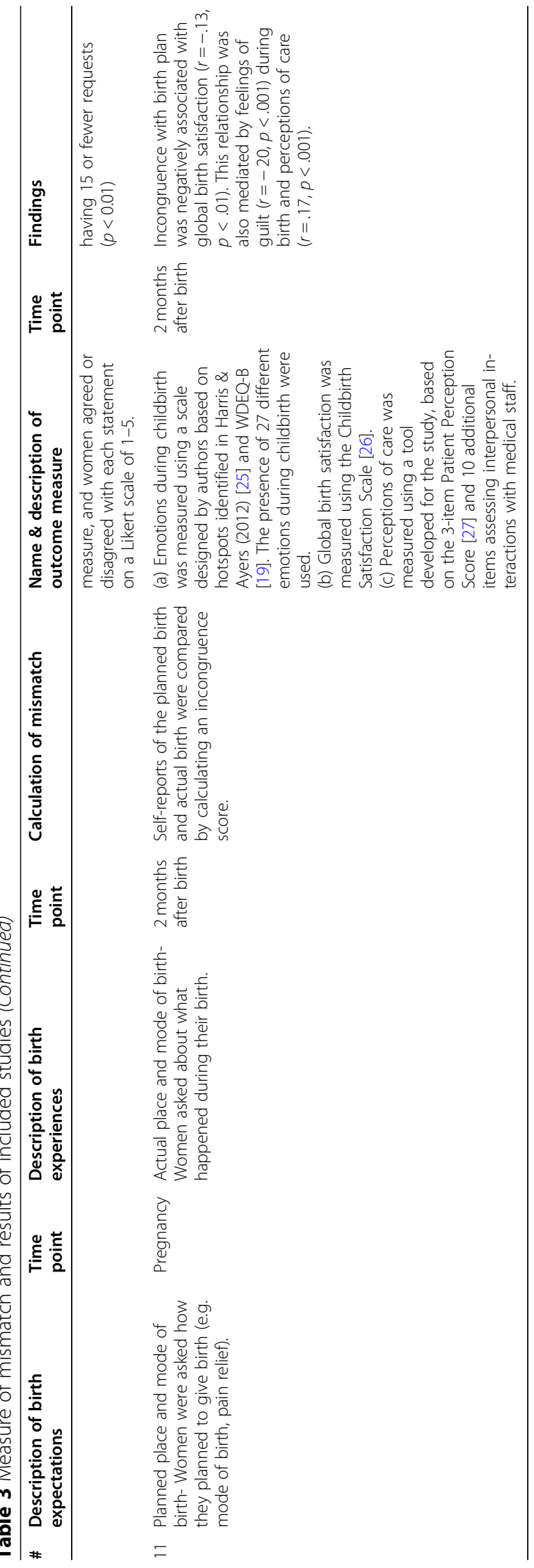


greater risk for severe FOC. This risk was not demonstrated for women who wanted a CS but had a vaginal birth.

Three studies looked at postnatal PTSD as an outcome of a mismatch between birth expectations and experiences. One study [47] showed that women who had a preference for a CS but did not receive one had significantly higher levels of PTSD compared to women who had no preference for a CS and did not receive one [47]. Furthermore, two studies [51, 52], found that more negative childbirth experiences than expected were associated with more PTSD symptoms after birth. However, both studies identified other factors that were also associated with the development of postnatal PTSD symptoms such as pain severity, powerlessness, history of sexual trauma, social support, medical interventions, length of labour [52], higher trait anxiety, greater antenatal depression scores and a less positive perception of care received during labour [51].

\section{Physical outcomes}

One study [46] used a health-related quality of life (HRQoL) measure [57] to evaluate the impact of a mismatch between birth expectations and experiences on physical health in women who had previously had a CS. HRQoL measures subjective influence of the health status (physical and emotional) on daily functioning and not quality of life per se [58]. The authors found that women who had a match between their preferred mode of birth and actual mode of birth had higher HRQoL scores three months after birth. More specifically, women who wanted a vaginal birth after caesarean (VBAC) and had one, had the highest HRQoL scores. In contrast, women who wanted a VBAC but had an elective repeated CS or an emergency repeated CS had lower HRQoL scores.

\section{Discussion}

This review aimed to understand the impact of a mismatch between labour/birth expectations and experiences on postnatal outcomes for the mother, her partner and her infant. The review identified 11 studies for inclusion. The majority of studies measured birth satisfaction or psychological symptoms as outcomes. Most studies were good quality, with only one rated as poor quality. Results from the review suggest that a mismatch between labour/birth expectations and experiences has a negative impact on women's satisfaction with birth and may increase the risk of women developing PTSD after birth. There was not enough evidence to draw conclusions about postnatal depression, FOC or physical wellbeing.

The finding that a mismatch between birth expectations and experiences is associated with lower birth satisfaction is supported by similar research that was not included in this review, due to not meeting inclusion criteria. For example, several studies were excluded because they did not calculate a mismatch between birth expectations and birth experiences, but instead used a correlation $(n=2)[59,60]$, did not calculate a mismatch $(n=2)$ [61, 62], or measured birth expectations and experiences at the same time [63, 64]. These studies also found that women who have a mismatch between birth expectations and experiences are less likely to rate their births as satisfying.

Results from the review also suggest that unmet expectations may increase women's risk of developing PTSD after birth. However, all three studies examining this found that multiple other risk factors, such as fear of childbirth, depression, pain severity, feelings of powerless, lack of adequate information provision and previous trauma increased the chances of women developing PTSD $[47,51,52]$. This is in line with previous research that has demonstrated several factors can contribute to women's risk of developing postnatal PTSD [39, 65-67]. This shows it is unlikely to be the unmet birth expectations themselves that lead to the development of postnatal PTSD, but a range of interrelated factors.

Results about the impact of unmet birth expectations on other psychological outcomes is less clear. Some studies suggested that unmet birth expectations are associated with psychological difficulties, such as depression [50], and FOC [48], whereas others found no relationship $[49,54,56]$. There are no clear patterns across the studies in terms of the number of women involved, the country of the study, the measure of the mismatch or the outcome measure used. This means it is not possible to hypothesise why some studies noted an association between unmet expectations and psychological outcomes and others did not. However, based on the results regarding birth satisfaction, it is possible that birth satisfaction might mediate the relationship between unmet expectations and psychological outcomes. It is also possible other factors may moderate the relationship (such as individual vulnerability e.g. previous trauma, interpersonal violence or obstetric risk).

\section{Implications for practice}

The results from this review suggest that women should have their desires relating to labour and birth listened to in order to improve birth satisfaction and potentially reduce the risk of PTSD. Birth satisfaction is a complex concept with many factors predicting it, such as time taken to hold baby, perceived threat to self, perceived threat to baby, birth type, perceived control [56] and the way women were treated by staff $[68,69]$. Furthermore, predictors such as communication and information provision during birth are related to PTSD [70]. Therefore, 
healthcare professionals can improve birth satisfaction and potentially reduce the risk of PTSD through a range of actions and sensitive, clear communication, involving women in decision making and listening to their needs [71-75].

\section{Implications for research}

The lack of prospective research carried out in this area makes conclusions difficult to draw. Measuring birth expectations during pregnancy, rather than after birth is more methodologically rigorous than retrospective measurement as it avoids recall bias. Furthermore, studies were variable in the methods used to measure a mismatch which also makes conclusions difficult to draw. Future research should focus on developing a standardised way of measuring this, to make cross-study comparisons easier. No prospective studies were identified that looked at the impact of a mismatch between birth expectations and experiences on birth partners, or women's children. Additionally, research on physical outcomes was minimal. Therefore, more prospective research is needed in these areas to examine the gaps in this knowledge.

\section{Limitations}

There are several limitations of this review that should be taken into consideration. For example, there are very few studies that evaluate the impact of a mismatch between birth expectations and experiences which makes conclusions difficult to draw. Most of the studies included evaluated the impact of a mismatch on birth satisfaction and psychological outcomes. Whilst the evidence is clearest for birth satisfaction and postnatal PTSD, the mixed results of the impact of a mismatch between birth expectations and experiences on other psychological outcomes suggests more prospective research is needed. Arguably, a limitation of the review methodology is the exclusion of studies where birth experiences and expectations were measured at the same time, or where a mismatch was not calculated. This may have meant some studies were missed. However, those studies that were identified and excluded supported the review findings [59-64]. Further, this decision meant that studies that were included avoided issues of recall bias and allowed a clear relationship between a birth experiences/ expectation mismatch and the outcome variable to be ascertained. This approach may have also contributed to the high-quality methodology of the majority of the studies included in the review.

\section{Conclusion}

Overall, this systematic review aimed to synthesise more methodologically rigorous evidence to understand whether unmet birth expectations are associated with adverse outcomes for women, their partners and their infants. The review found that the relationship between unmet birth expectations and depression/FOC and physical outcomes is not clear and more research is needed. However, the results from this review did identify that a mismatch between birth expectations and experiences is associated with birth satisfaction and may increase the risk of developing postnatal PTSD. Further prospective research is needed to identify examine gaps in knowledge and provide standardised methods of measuring childbirth expectations-experiences mismatch. To ensure women's expectations are met, and therefore experience a satisfying birth experience, maternity providers should provide sensitive care, which acknowledges women's needs and preferences, is based on open and clear communication, is delivered as early in pregnancy as possible, and enables women to make their own decisions about their care.

\section{Supplementary Information}

The online version contains supplementary material available at https://doi. org/10.1186/s12884-021-03898-z.

\section{Additional file 1}

\section{Acknowledgements}

This study was from the EU funded COST action CA18211: DEVOTION: Perinatal Mental Health and Birth-Related Trauma: Maximising best practice and optimal outcomes where Susan Ayers, Annick Bogaerts, Rita Borg Xuereb, Ljiljana Jeličić and Paulina Pawlicka are management committee members and/or part of working group 3.2. We also gratefully acknowledge the input of other members of this working group: Isabel Soares, Mirjana Sovilj and Stef Savona Ventura.

COST action CA18211:DEVoTION team Consortium Members

Isabel Soares ${ }^{1}$, Mirjana Sovilj ${ }^{2}$ and Stef Savona Ventura ${ }^{3}$

1. School of Psychology, University of Minho, Portugal

2. Institute for Experimental Phonetics and Speech Pathology," Djordje Kostic", Belgrade, Serbia

3. Mater Dei Hospital, Malta

\section{Authors' contributions}

The management committee of the working group ( $S A, A B, L, P P, R B X$ ) conceptualised the idea. RW carried out the searches, contributed to title and abstract screening, carried out full text screening, data analysis, creation of figures, data interpretation and write up of manuscript. SA conceptualised the idea, helped to refine search terms, provided support with data analysis and provided detailed feedback of the manuscript. $A B, L J$ conceptualised the idea, provided support with data analysis and provided detailed feedback of the manuscript. PP conceptualised the idea, double coded the quality assessment of the included reviews, provided support with data analysis and provided detailed feedback of the manuscript. SVH double coded the quality assessment of the included reviews and provided detailed feedback on the manuscript. NU double screened title and abstract and full texts. RBX provided support with data analysis and provided detailed feedback on the manuscript. NK helped to refine search terms and this project formed part of her master's project. The author(s) read and approved the final manuscript.

\section{Funding}

This study was from the EU funded COST action CA18211: DEVOTION: Perinatal Mental Health and Birth-Related Trauma: Maximising best practice and optimal outcomes where Susan Ayers, Annick Bogaerts, Ljiljana Jeličić and Paulina Pawlicka are management committee members and part of working group 3.2 . 


\section{Availability of data and materials}

Data sharing is not applicable to this article as no datasets were generated or analysed during the current study.

\section{Declarations}

\section{Ethics approval and consent to participate}

Not applicable - systematic review.

\section{Consent for publication}

Not applicable - systematic review.

\section{Competing interests}

The authors declare that they have no competing interests.

\section{Author details}

${ }^{1}$ Centre for Maternal and Child Health Research, City, University of London, London EC1V OHB, UK. ${ }^{2}$ KU Leuven, Department of Development and Regeneration, Research Unit Women and Child, B-3000 Leuven, Belgium. ${ }^{3}$ Faculty of Medicine and Health Sciences, Centre for Research and Innovation Care (CRIC), University of Antwerp, Antwerp, Belgium. ${ }^{4}$ Faculty of Health, University of Plymouth, Plymouth, Devon PL4 8AA, UK. ${ }^{5}$ Cognitive Neuroscience Department, Institute for Research and Development "Life Activities Advancement Center", Belgrade, Serbia. 'Department of Speech, Language and Hearing Sciences, Institute for Experimental Phonetics and Speech Pathology, Belgrade, Serbia. ${ }^{7}$ Department of Social Sciences, Institute of Psychology, University of Gdańsk, Gdańsk, Poland. ${ }^{8}$ Research and Expertise, Resilient People, University College Leuven-Limburg, Diepenbeek, Belgium. ${ }^{9}$ Department of Midwifery, Faculty of Health Sciences, University of Malta, Msida, Malta.

Received: 17 February 2021 Accepted: 20 May 2021

Published online: 02 July 2021

\section{References}

1. O'Brien D, Casey M, Butler MM. Women's experiences of exercising informed choices as expressed through their sense of self and relationships with others in Ireland: a participatory action research study. Midwifery. 2018; 65:58-66. https://doi.org/10.1016/j.midw.2018.07.006.

2. Parratt J. The impact of childbirth experiences on women's sense of self: a review of the literature. Aust J Midwifery. 2002;15(4):10-6. https://doi.org/1 0.1016/S1031-170X(02)80007-1.

3. Bai G, Korfage IJ, Mautner E, Raat H. Determinants of maternal health-related quality of life after childbirth: the generation R study. Int J Environ Res Public Health. 2019;16(18):3231. https://doi.org/10.3390/ijerph16183231.

4. Rowlands IJ, Redshaw M. Mode of birth and women's psychological and physical wellbeing in the postnatal period. BMC Pregnancy Childbirth. 2012; 12(1):1-1. https://doi.org/10.1186/1471-2393-12-138.

5. Karlström A, Nystedt A, Hildingsson I. The meaning of a very positive birth experience: focus groups discussions with women. BMC Pregnancy Childbirth. 2015;15(1):1-8. https://doi.org/10.1186/s12884-015-0683-0.

6. Olza I, Leahy-Warren P, Benyamini Y, Kazmierczak M, Karlsdottir SI, Spyridou A, et al. Women's psychological experiences of physiological childbirth: a meta-synthesis. BMJ Open. 2018;8(10):e020347. https://doi.org/10.1136/ bmjopen-2017-020347.

7. McGrath P, Phillips E, Vaughan G. Speaking out! Qualitative insights on the experience of mothers who wanted a vaginal birth after a birth by cesarean section. Patient. 2010;3(1):25-32. https://doi.org/10.2165/11318810000000000-00000.

8. Gottvall K, Waldenström U. Does a traumatic birth experience have an impact on future reproduction? BJOG An Int J Obstet Gynaecol. 2002;109(3): 254-60. https://doi.org/10.1111/j.1471-0528.2002.01200.x.

9. Thomson G, Downe S. Emotions and support needs following a distressing birth: scoping study with pregnant multigravida women in north-West England. Midwifery. 2016;40:32-9. https://doi.org/10.1016/j. midw.2016.06.010

10. Wijma K, Söderquist J, Wijma B. Posttraumatic stress disorder after childbirth: a cross sectional study. J Anxiety Disord. 1997;11(6):587-97. https://doi.org/1 0.1016/S0887-6185(97)00041-8.

11. Michels A, Kruske S, Thompson R. Women's postnatal psychological functioning: the role of satisfaction with intrapartum care and the birth experience. J Reprod Infant Psychol. 2013;31(2):172-82. https://doi.org/10.1 080/02646838.2013.791921.

12. Murray L, Fiori-Cowley A, Hooper R, Cooper P. The impact of postnatal depression and associated adversity on early mother-infant interactions and later infant outcome. Child Dev. 1996;67(5):2512-26. https://doi.org/10.1111/ j.1467-8624.1996.tb01871.x.

13. Beck CT. The effects of postpartum depression on child development: a meta-analysis. Arch Psychiatr Nurs. 1998;12(1):12-20. https://doi.org/10.1016/ S0883-9417(98)80004-6.

14. O'Donnell M, Maclean MJ, Sims S, Morgan VA, Leonard H, Stanley FJ. Maternal mental health and risk of child protection involvement: mental health diagnoses associated with increased risk. J Epidemiol Community Health. 2015;69(12):1175-83. https://doi.org/10.1136/jech-2014-205240.

15. Rees S, Channon S, Waters CS. The impact of maternal prenatal and postnatal anxiety on children's emotional problems: a systematic review. Eur Child Adolesc Psychiatry. 2019;28(2):257-80. https://doi.org/10.1007/s00787018-1173-5.

16. Parfitt Y, Pike A, Ayers S. Infant developmental outcomes: a family systems perspective. Infant Child Dev. 2014;23(4):353-73. https://doi.org/10.1002/ icd.1830.

17. O'Donnell KJ, Glover $\mathrm{V}$, Barker ED, O'Connor TG. The persisting effect of maternal mood in pregnancy on childhood psychopathology. Dev Psychopathol. 2014;26(2):393-403. https://doi.org/10.1017/S0954579414 000029

18. Liu Y, Kaaya S, Chai J, McCoy DC, Surkan PJ, Black MM, et al. Maternal depressive symptoms and early childhood cognitive development: a metaanalysis. Psychol Med. 2017;47(4):680-9. https://doi.org/10.1017/S003329171 600283X.

19. Netsi E, Pearson RM, Murray L, Cooper P, Craske MG, Stein A. Association of persistent and severe postnatal depression with child outcomes. JAMA Psychiatry. 2018;75(3):247-53. https://doi.org/10.1001/jamapsychiatry.2017.4363.

20. Oyetunji A, Chandra P. Postpartum stress and infant outcome: a review of current literature. Psychiatry Res. 2020;284:112769. https://doi.org/10.1016/j. psychres.2020.112769.

21. Letourneau N, Leung B, Ntanda H, Dewey D, Deane AJ, Giesbrecht GF. Maternal and paternal perinatal depressive symptoms associate with 2-and 3-year-old children's behaviour: findings from the APrON longitudinal study. BMC Pediatr. 2019;19(1):1-3. https://doi.org/10.1186/s12887-019-1775-1.

22. Prenoveau JM, Craske MG, West V, Giannakakis A, Zioga M, Lehtonen A, et al. Maternal postnatal depression and anxiety and their association with child emotional negativity and behavior problems at two years. Dev Psychol. 2017:53(1):50-62. https://doi.org/10.1037/dev0000221.

23. Glasheen C, Richardson GA, Fabio A. A systematic review of the effects of postnatal maternal anxiety on children. Arch Womens Ment Health. 2010; 13(1):61-74. https://doi.org/10.1007/s00737-009-0109-y.

24. Ayers S, Pickering AD. Do women get posttraumatic stress disorder as a result of childbirth? A prospective study of incidence. Birth. 2001;28(2):1118. https://doi.org/10.1046/j.1523-536X.2001.00111.x.

25. Beaton J, Gupton A. Childbirth expectations: a qualitative analysis. Midwifery. 1990;6(3):133-9. https://doi.org/10.1016/S0266-6138(05)80170-6.

26. Beebe KR, Humphreys J. Expectations, perceptions, and Management of Labor in Nulliparas prior to hospitalization. J Midwifery Womens Health. 2006;51(5):347-53. https://doi.org/10.1016/j.jmwh.2006.02.013.

27. Gibbins J, Thomson AM. Women's expectations and experiences of childbirth. Midwifery. 2001;17(4):302-13. https://doi.org/10.1054/midw.2 001.0263 .

28. Martin DK, Bulmer SM, Pettker CM. Childbirth expectations and sources of information among low- and moderate-income nulliparous pregnant women. J Perinat Educ. 2013;22(2):103-12. https://doi.org/10.1891/1 058-1243.22.2.103.

29. Singh D, Newburn M. Access to maternity information and support: the experiences and needs of women before and after giving birth. London: National Childbirth Trust; 2000.

30. Davis-Floyd RE. Birth as an American Rite of Passage (2nd edition). Berkley: University of California Press; 2004.

31. Preis $H$, Pardo J, Peled $Y$, Benyamini $Y$. Changes in the basic birth beliefs following the first birth experience: self-fulfilling prophecies? PLOS One. 2018;13(11):e0208090. https://doi.org/10.1371/journal.pone.0208090.

32. Kornelsen J. Essences and imperatives: an investigation of technology in childbirth. Soc Sci Med. 2005;61(7):1495-504. https://doi.org/10.1016/j. socscimed.2005.03.007 
33. Miller AC, Shriver TE. Women's childbirth preferences and practices in the United States. Soc Sci Med. 2012;75(4):709-16. https://doi.org/10.1016/j. socscimed.2012.03.051.

34. Mei JY, Afshar Y, Gregory KD, Kilpatrick SJ, Esakoff TF. Birth plans: what matters for birth experience satisfaction. Birth. 2016;43(2):144-50. https://doi. org/10.1111/birt.12226

35. Divall B, Spiby H, Nolan M, Slade P. Plans, preferences or going with the flow: an online exploration of women's views and experiences of birth plans. Midwifery. 2017:54:29-34. https://doi.org/10.1016/j.midw.2017.07.020.

36. Welsh JV, Symon AG. Unique and proforma birth plans: a qualitative exploration of midwives' experiences. Midwifery. 2014;30(7):885-91. https:// doi.org/10.1016/j.midw.2014.03.004

37. Aragon M, Chhoa E, Dayan R, Kluftinger A, Lohn Z, Buhler K. Perspectives of expectant women and health care providers on birth plans. J Obstet Gynaecol Can. 2013;35(11):979-85. https://doi.org/10.1016/S1701-2163(15)30785-4.

38. Sato $S$, Umeno $Y$. The relationship between the recognition of postpartum mothers' birth plan and the degree of satisfaction with delivery. J Jpn Acad Midwifery. 2011;25(1):27-35.

39. Waldenström U, Hildingsson I, Rubertsson C, Rådestad I. A negative birth experience: prevalence and risk factors in a national sample. Birth. 2004; 31(1):17-27. https://doi.org/10.1111/j.0730-7659.2004.0270.x

40. Hauck Y, Fenwick J, Downie J, Butt J. The influence of childbirth expectations on Western Australian women's perceptions of their birth experience. Midwifery. 2007;23(3):235-47. https://doi.org/10.1016/j.midw.2006.02.002.

41. Procter S, Hollins Martin CJ, Larkin D, Martin CR. Woman's experience of childbirth: qualitative analysis from data derived from the 30-item birth satisfaction scale. Sci Pages Nurs. 2017;1(1):10-7.

42. Matejić B, Milićević MT, Vasić V, Djikanović B. Maternal satisfaction with organized perinatal care in Serbian public hospitals. BMC Pregnancy Childbirth. 2014;14(1):1-9. https://doi.org/10.1186/1471-2393-14-14.

43. Moher D, Shamseer L, Clarke M, Ghersi D, Liberati A, Petticrew M, et al. Preferred reporting items for systematic review and meta-analysis protocols (PRISMA-P) 2015 statement. Syst Rev. 2015;4(1):1-9. https://doi.org/10.1186/2046-4053-4-1.

44. The Joanna Briggs Institute. Critical appraisal tools: Checklist for Cohort Studies. 2017. https://jbi.global/critical-appraisal-tools

45. Lucas PJ, Baird J, Arai L, Law C, Roberts HM. Worked examples of alternative methods for the synthesis of qualitative and quantitative research in systematic reviews. BMC Med Res Methodol. 2007;7(1):4. https://doi.org/1 0.1186/1471-2288-7-4.

46. Fobelets M, Beeckman K, Buyl R, Healy P, Grylka-Baeschlin S, Nicoletti J, et al. Preference of birth mode and postnatal health related quality of life after one previous caesarean section in three European countries. Midwifery. 2019;79:102536. https://doi.org/10.1016/j.midw.2019.102536.

47. Garthus-Niegel S, von Soest T, Knoph C, Simonsen TB, Torgersen L, Eberhard-Gran M. The influence of women's preferences and actual mode of delivery on post-traumatic stress symptoms following childbirth: a population-based, longitudinal study. BMC Pregnancy Childbirth. 2014;14(1): 1-0. https://doi.org/10.1186/1471-2393-14-191

48. Sluijs AM, Wijma K, Cleiren MPHD, van Lith JMM, Wijma B. Preferred and actual mode of delivery in relation to fear of childbirth. J Psychosom Obstet Gynecol. 2020;41(4):266-74. https://doi.org/10.1080/01 67482X.2019.1708319.

49. Tanglakmankhong K, Perrin N. Childbirth expectations and childbirth experiences among Thai pregnant women. Portland: Oregon Health \& Science University, School of Nursing; 2010.

50. Houston KA, Kaimal AJ, Nakagawa S, Gregorich SE, Yee LM, Kuppermann M. Mode of delivery and postpartum depression: the role of patient preferences. Am J Obstet Gynecol. 2015;212(2):229-e1. https://doi.org/10.1 016/j.ajog.2014.09.002.

51. Verreault N, Da Costa D, Marchand A, et al. PTSD following childbirth: a prospective study of incidence and risk factors in Canadian women. J Psychosom Res. 2012;73(4):257-63. https://doi.org/10.1016/j.jpsychores.2012.07.010.

52. Soet JE, Brack GA, Dilorio C. Prevalence and predictors of women's experience of psychological trauma during childbirth. Birth. 2003;30(1):3646. https://doi.org/10.1046/j.1523-536X.2003.00215.x.

53. Wijma K, Wijma B, Zar M. Psychometric aspects of the W-DEQ; a new questionnaire for the measurement of fear of childbirth. J Psychosom Obstet Gynaecol. 1998;19(2):84-97. https://doi.org/10.3109/01674829809048501.

54. Phillipson-Price A. Expectancy and the experience of childbirth: the effect of the relationship on postpartum affect. Montreal: McGill University; 1982.
55. Preis $H$, Lobel $M$, Benyamini $Y$. Between expectancy and experience: testing a model of childbirth satisfaction. Psychol Women Q. 2019:43(1):105-17. https://doi.org/10.1177/0361684318779537.

56. Deluca RS, Lobel M. Diminished control and unmet expectations: testing a model of adjustment to unplanned cesarean delivery. Anal Soc Issues Public Policy. 2014;14(1):183-204. https://doi.org/10.1111/asap.12040.

57. Brazier JE, Harper R, Jones NMB, et al. Validating the SF-36 health survey questionnaire: new outcome measure for primary care. Br Med J. 1992; 305(6846):160-4. https://doi.org/10.1136/bmj.305.6846.160.

58. Miniszewska J, Chodkiewicz J, Ograczyk-Piotrowska A, Zalewska-Janowska A. Life satisfaction and health related quality of life - the same or a different construct? A survey in psoriasis patients. Health Psychol Rep. 2020;8(3):21927. https://doi.org/10.5114/hpr.2020.95909.

59. Fair CD, Morrison TE. The relationship between prenatal control, expectations, experienced control, and birth satisfaction among primiparous women. Midwifery. 2012;28(1):39-44. https://doi.org/10.1016/j.midw.2010.10.013.

60. Kuo SC, Lin KC, Hsu CH, et al. Evaluation of the effects of a birth plan on Taiwanese women's childbirth experiences, control and expectations fulfilment: A randomised controlled trial. Int J Nurs Stud. 2010. https://doi. org/10.1016/j.jijnurstu.2009.11.012.

61. Goodman P, Mackey MC, Tavakoli AS. Factors related to childbirth satisfaction. J Adv Nurs. 2004;47(7):806-14. https://doi.org/10.1111/j.1365-2648.2003.02981.x.

62. Green JM, Coupland VA, Kitzinger JV. Expectations, experiences, and psychological outcomes of childbirth: a prospective study of 825 women. Birth. 1990;17(1):15-24. https://doi.org/10.1111/j.1523-536X.1990.tb00004.X.

63. Christiaens W, Bracke P. Assessment of social psychological determinants of satisfaction with childbirth in a cross-national perspective. BMC Pregnancy Childbirth. 2007;7(1):1-2. https://doi.org/10.1186/1471-2393-7-26.

64. Jacoby A. Women's preferences for and satisfaction with current procedures in childbirth-findings from a national study. Midwifery. 1987;3(3):117-24. https://doi.org/10.1016/S0266-6138(87)80022-0.

65. Dikmen-Yildiz P, Ayers S, Phillips L. Factors associated with post-traumatic stress symptoms (PTSS) 4-6 weeks and 6 months after birth: a longitudinal population-based study. J Affect Disord. 2017;221:238-45. https://doi.org/1 0.1016/j.jad.2017.06.049.

66. Grekin R, O'Hara MW. Prevalence and risk factors of postpartum posttraumatic stress disorder: a meta-analysis. Clin Psychol Rev. 2014;34(5): 389-401. https://doi.org/10.1016/j.cpr.2014.05.003.

67. Söderquist J, Wijma B, Thorbert G, Wijma K. Risk factors in pregnancy for posttraumatic stress and depression after childbirth. BJOG An Int J Obstet Gynaecol. 2009;1 16(5):672-80. https://doi.org/10.1111/j.1471-0528.2008.02083.x.

68. Lomas J, Dore S, Enkin M, Mitchell A. The labor and delivery satisfaction index: the development and evaluation of a soft outcome measure. Birth. 1987;14(3):125-9. https://doi.org/10.1111/j.1523-536X.1987.tb01472.x.

69. Mackey MC. Women's evaluation of the labor and delivery experience. Nursingconnections. 1998;11(3):19-32.

70. Reed R, Sharman R, Inglis C. Women's descriptions of childbirth trauma relating to care provider actions and interactions. BMC Pregnancy Childbirth. 2017;17(1):1-0. https://doi.org/10.1186/s12884-016-1197-0.

71. Lewis L, Hauck YL, Ronchi F, Crichton C, Waller L. Gaining insight into how women conceptualize satisfaction: Western Australian women's perception of their maternity care experiences. BMC Pregnancy Childbirth. 2016;16(1):19. https://doi.org/10.1186/s12884-015-0759-x.

72. Konheim-Kalkstein YL, Miron-Shatz T, Israel LJ. How women evaluate birth challenges: analysis of web-based birth stories. JMIR Pediatr Parent. 2018; 1(2):e12206. https://doi.org/10.2196/12206.

73. Srivastava A, Avan Bl, Rajbangshi P, Bhattacharyya S. Determinants of women's satisfaction with maternal health care: a review of literature from developing countries. BMC Pregnancy Childbirth. 2015;15(1):1-2. https://doi. org/10.1186/s12884-015-0525-0.

74. Hodnett ED. Pain and women's satisfaction with the experience of childbirth: a systematic review. Am J Obstet Gynecol. 2002;186(5):S160-72. https://doi.org/10.1067/mob.2002.121141.

75. Chabbert M, Panagiotou D, Wendland J. Predictive factors of women's subjective perception of childbirth experience: a systematic review of the literature. J Reprod Infant Psychol. 2021;39(1):43-66. https://doi.org/10.1080/ 02646838.2020.1748582.

\section{Publisher's Note}

Springer Nature remains neutral with regard to jurisdictional claims in published maps and institutional affiliations. 